\title{
BEHAVIOUR OF COMPOSITE STEEL TUBES INFILLED WITH FRC UNDER MONOTONIC LOADING
}

\author{
Sudha $K^{1}$, Khalid Nayaz Khan ${ }^{2}$, N.S.Kumar ${ }^{3}$

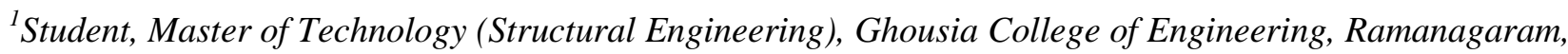 \\ Karnataka, India \\ ${ }^{2}$ Associate Professor, Ghousia College of Engineering, Ramanagaram, Karnataka, India \\ ${ }^{3}$ Professor \& Director $(R \& D)$, Ghousia College of Engineering, Ramanagaram, Karnataka, India
}

\begin{abstract}
In this paper, experimental investigation on the behavior of concrete filled steel tube infilled with Polypropylenefibre (PPF) under monotonic loading is carried out. Here the strength of 54 Specimens was tested. The parameters varied in this study are 1) percentage of PPF $(0 \%, 0.2 \%, 0.3 \%, 0.4 \%)$ 2) Diameter of the hollow circular steel tubes $(33.7 \mathrm{~mm} \mathrm{and} 42.4 \mathrm{~mm}) 3) \mathrm{L} / \mathrm{D}$ ratios(10,12 and 14) and 4) Grades of concrete(M20 and M30). The thickness of steel tubes is 3.2mm which remains same for all the specimens. Polypropylene fiber concrete filled steel columns exhibit higher strength than steel tubes filled with conventional concrete. The results show that load carrying capacity increased with increase in percentage of polypropylene. it was observed that the load carrying capacity of the steel tube decreased as the D/t ratio increased. The experimental results were compared with theoretical results and analysis results using ABAQUS software
\end{abstract}

Keywords: Concrete Filled Steel Tube (CFST), Polypropylene Fibre (PPF)

\section{INTRODUCTION}

CFST is a composite structural member, which resists the applied loads through the composite action of concrete and steel. CFST increases the ultimate load bearing of the member and it is also gives aesthetically pleasing. Since the steel confines concrete bucking resistance of the CFST member increases. The restrained effect of concrete present in the CFST tube helps in delaying the local buckling of the steel tube and also prevents the failure of the member. The confinement effect of the steel tube increases the strength of and also strength deterioration is not that serious because concrete does not spall due to the confinement. Having listed all the advantages, however the major disadvantage of a composite column is the exposure of tube to the environmental effects (such as heat, cold, UV etc). For steel tubes, this raises concerns related to susceptibility to corrosion and fire safety. CFST column posses high strength, high ductility more energy absorption capacity. The ultimate load bearing capacity and resistance to bending, compression and shear are all better than that of the reinforced concrete. On other hand Use of thin walled steel tubes in construction reduces the cost of construction significantly The reduction of the steel tube thickness in thin-walled CFST columns has the potential to significantly reduce construction costs. Concrete filled steel tubular (CFST) column, comprising a hollow steel tube infilled with concrete with or without additional reinforcements or steel section, has been widely used in high rise building construction.

\subsection{Types of CFST Columns}

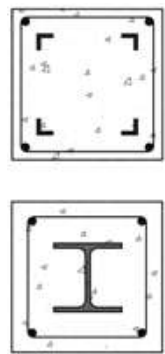

a)
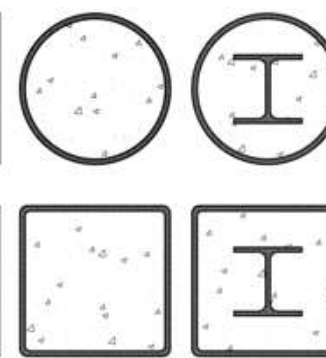

b)

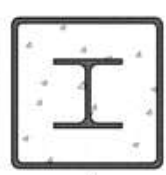

c)
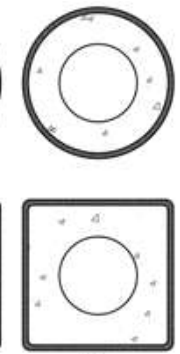

d)
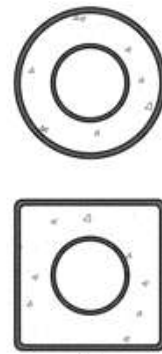

e)
Fig 1: Types of concrete filled steel tubular cross sections

\subsection{Materials and Methodology}

\subsubsection{Cement}

In this experiment 53 grades ordinary Portland cement (OPC) was used for all concrete mixes. The properties of cement used are as shown in table.1.

Table 1 properties of Cement

\begin{tabular}{|l|l|l|}
\hline Sl. no & properties & value \\
\hline 1 & Brand of Cement & Zuari \\
\hline 2 & Type of Cement & 53 grade \\
\hline 3 & Specific gravity & 3.14 \\
\hline
\end{tabular}

\subsubsection{Fine Aggregates}

For this investigation manufactured sand is used as fine aggregates free from deleterious materials. The properties of Fine aggregates used are tabulated in table.2. 
Table 2: Properties of fine aggregates

\begin{tabular}{|l|l|l|}
\hline Sl. No & properties & value \\
\hline 1 & Specific gravity & 2.50 \\
\hline 2 & Fineness modulus & 3.015 \\
\hline 3 & Grading of sand & Zone II \\
\hline
\end{tabular}

\subsubsection{Coarse Aggregates}

The coarse aggregates used for this experiment is $12 \mathrm{~mm}$ size which is free from deleterious materials. The physical properties are as given in table.3.

Table 3: Properties of coarse aggregates

\begin{tabular}{|l|l|l|}
\hline Sl. No & properties & value \\
\hline 1 & Specific gravity & 2.67 \\
\hline 2 & Fineness modulus & 2.24 \\
\hline
\end{tabular}

\subsubsection{Water}

Clean portable tab water available from library is used for the experiments.

\subsubsection{Polypropylene}

Polypropylene is one of the cheapest and more available fibers. It has high resistant to chemical attacks and high melting point about 165 degree centigrade. It can withstand a working temp 100 degree centigrade.
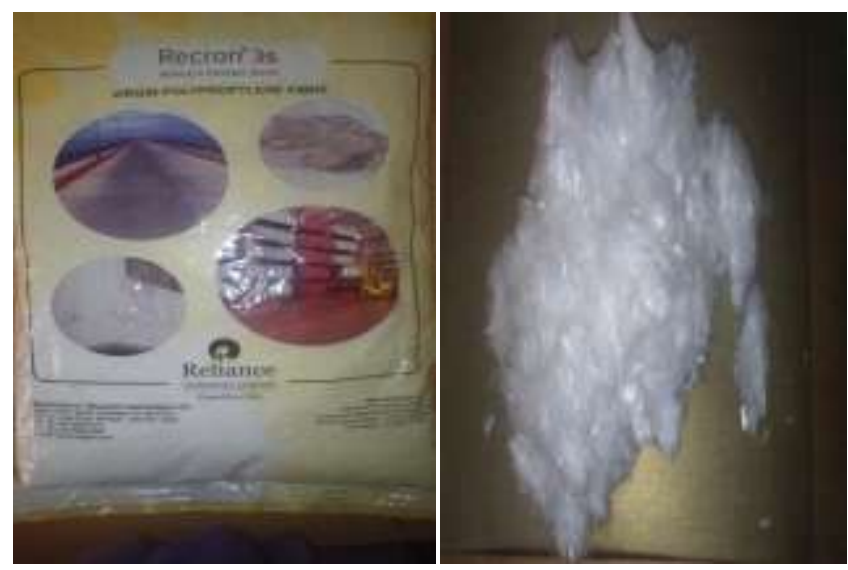

Fig 2: Polypropylene Fiber

\subsection{Concrete Mix Design}

The Mix Proportions for M20 Cement: A: CA 1:1.5:3 The Mix Proportions for M20 Cement: A: CA 1:1.5:2.5

\subsection{Compressive Strength After 28 Days}

Table 4: Compressive Strength after 28 days

\begin{tabular}{|c|c|c|c|c|c|}
\hline Sl no & Mix designation & \% of PPF & $\mathrm{C} / \mathrm{S}$ Area of cube in $\mathrm{mm}^{2}$ & Load in Tons & $\begin{array}{l}28 \text { days } \\
\text { Compressive } \\
\text { strength in } \mathrm{N} / \mathrm{mm}^{2}\end{array}$ \\
\hline 1 & \multirow{4}{*}{ M20 } & $0 \%$ & 225 & 51.5 & 22.5 \\
\hline 2 & & $0.2 \%$ & 225 & 62 & 27.05 \\
\hline 3 & & $0.3 \%$ & 225 & 65.5 & 28.6 \\
\hline 4 & & $0.4 \%$ & 225 & 69 & 30.05 \\
\hline 5 & \multirow{4}{*}{ M30 } & $0 \%$ & 225 & 73.2 & 31.95 \\
\hline 6 & & $0.2 \%$ & 225 & 78 & 34.06 \\
\hline 7 & & $0.3 \%$ & 225 & 81.5 & 35.5 \\
\hline 8 & & $0.4 \%$ & 225 & 85.5 & 37.28 \\
\hline
\end{tabular}

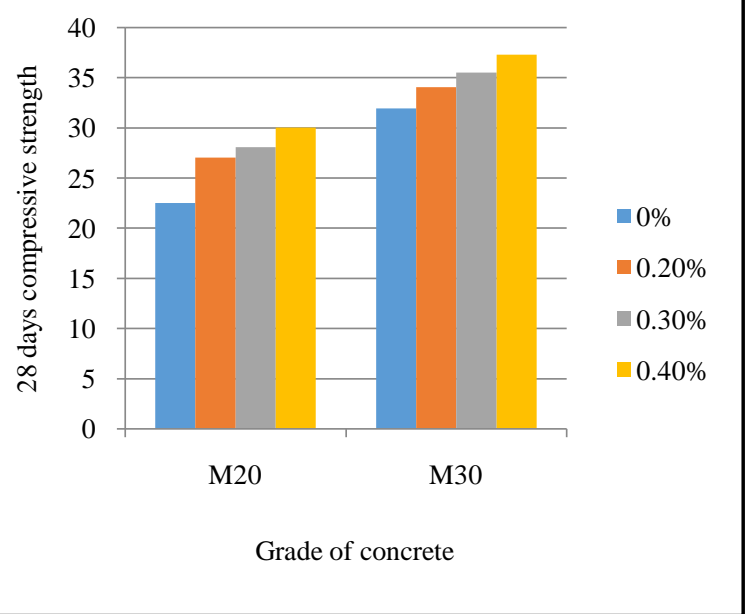

Chart 1: Variation of Compressive Strength for M20 and M30 Grade of Concrete

\subsection{Preparing Concrete Mix}

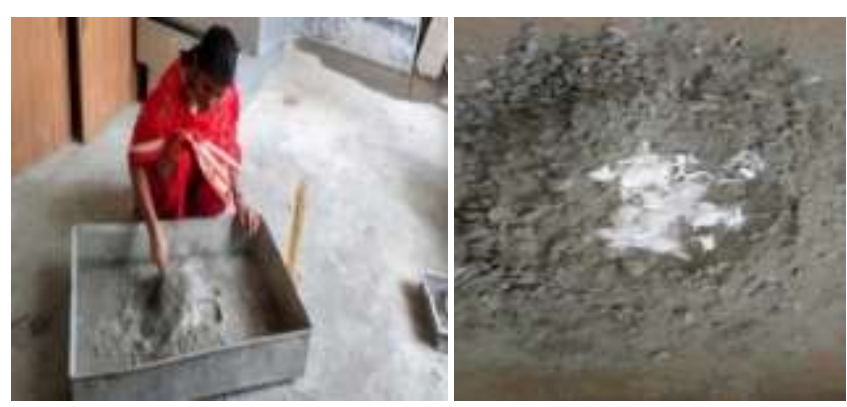

Fig 3: preparing concrete mix and adding fiber to concrete

\subsection{Steel Tube}

Circular steel tubes of yield strength310Mpa from Tata steel company were used 
Table 5: Circular tubes size and corresponding L/D ,D/T ratios for yield strength 310 grade

\begin{tabular}{|l|l|l|l|l|l|}
\hline $\begin{array}{l}\text { SL } \\
\text { NO }\end{array}$ & $\begin{array}{l}\text { Dia } \\
(\mathbf{m m})\end{array}$ & $\begin{array}{l}\mathbf{t} \\
(\mathbf{m m})\end{array}$ & $\begin{array}{l}\text { L/D } \\
\text { ratio }\end{array}$ & $\begin{array}{l}\text { Length } \\
(\mathbf{m m})\end{array}$ & $\begin{array}{l}\mathbf{D} / \mathbf{T} \\
\text { ratio }\end{array}$ \\
\hline 1 & 33.7 & 3.2 & 10 & 337 & 10.53 \\
\hline 2 & 33.7 & 3.2 & 12 & 405 & 10.53 \\
\hline 3 & 33.7 & 3.2 & 14 & 472 & 10.53 \\
\hline 4 & 42.4 & 3.2 & 10 & 424 & 13.25 \\
\hline 5 & 42.4 & 3.2 & 12 & 510 & 13.25 \\
\hline 6 & 42.4 & 3.2 & 14 & 594 & 13.25 \\
\hline
\end{tabular}

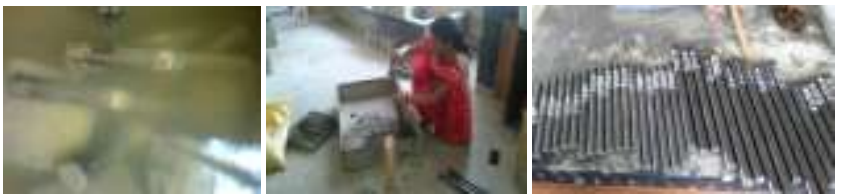

Fig 5: filling of steel tube with concrete and placing for curing

\section{EXPERIMENTAL SETUP:}

The tests were conducted using a 2000 ton capacity monotonic loading machine placing the specimen in the testing machine and geometry of the specimens are as shown in Fig. 1 to 3. The specimen was placed between the bearing plates in such a manner that the upper bearing plates was directly in line with the lower plate and the bearing plates extend at least $25 \mathrm{~mm}$ from each end of the specimen. The columns were placed on smooth plates at both ends. Care was taken to ensure that truly axial load was applied to each of the columns. Plumb bob and Theodolite has been employed to place the specimen truly vertical and hence load the specimen concentrically as shown in Fig. 4.

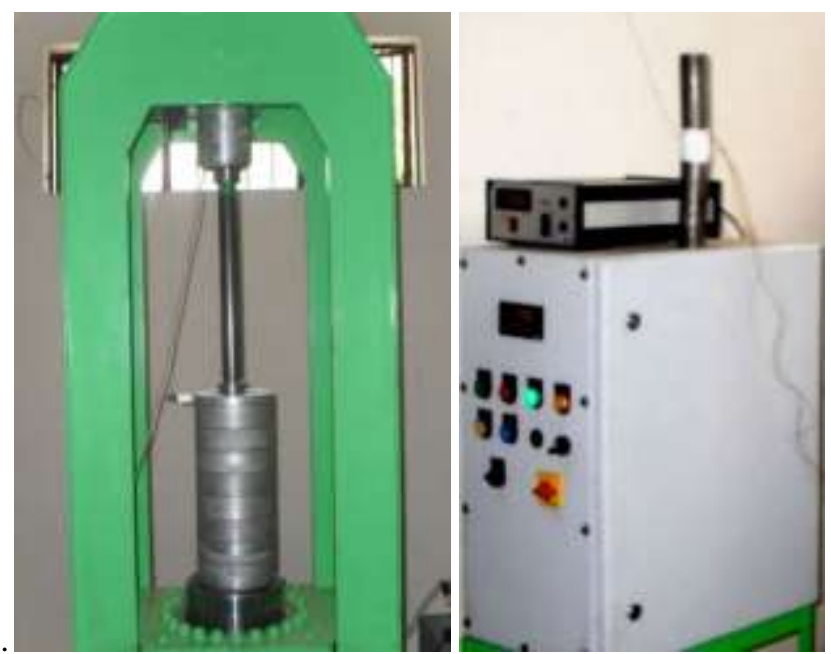

Fig 6: 200 Ton Capacity Hydraulic Monotonic Loading Machine

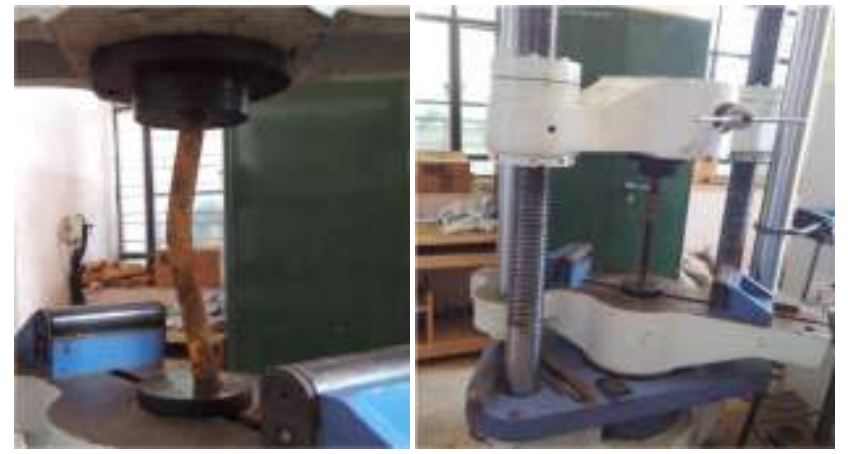

Fig 7: CFST column before and after applying load

\section{THEORETICAL FORMULAE \&} CALCULATION

\subsection{EC 4 - Euro Code}

EC-4 considers confinement effects of CFST member. This code is used to check ultimate load capacity. For circular column, where $\lambda$ is defined as

$$
\begin{aligned}
& \lambda=\frac{\sqrt{\text { Asfs }+0.85 A c f c}}{\mathrm{Ncr}} \\
& \mathrm{N}_{\mathrm{cr}}=\frac{(\text { EsIs }+ \text { EcIc }) \Pi^{2}}{L^{2}} \\
& \eta 1=4.9-18.5 \lambda+17 \lambda^{2}+1 \\
& \text { п2 }=0.25(3.2 \lambda) \\
& \mathrm{P}_{\mathrm{u}}=A s f s^{\mathrm{n}} 2+\operatorname{Acfc}\left(1+\mathrm{\eta} 1 \frac{t f s}{d f c}\right)
\end{aligned}
$$

\subsection{ACI 318-1999: American Concrete Institute-}

\section{Building Code Requirements for Structural}

\section{Concrete}

The ultimate load, $\mathrm{Pu}$ is given by equation

$$
\mathrm{Pu}=\mathrm{AsFs}+0.85 \mathrm{AcFc}
$$

\subsection{BS 5400 (British Standards)}

BS 5400 is based on limit state design and ultimate load carrying capacity $(\mathrm{Pu})$ is given by the equation

$$
\mathrm{NBS}=0.91 \text { As fy }+0.45 \text { Ac Fc }
$$

\subsection{CCECS Methods (China Engineering and}

\section{Construction Specification)}

$$
\mathrm{N}_{\text {CESS }}=\phi 1 . \phi 2 \cdot \mathrm{N}_{0}
$$

$\phi 1=1$ for $(\mathrm{L} / \mathrm{D}) \leq 4$

$\phi 1=1-0.115 L / D\left(\sqrt{ }\left(\frac{L}{D}\right)-4\right)$ for $(\mathrm{L} / \mathrm{D})>4$

$\phi 1$ and $\phi 2$ are reduction factors

$\mathrm{N}_{0}=\mathrm{fc} A \mathrm{c}+\mathrm{fs}$ As + SQRT ((fcAc). (FsAs)) 
Table 3 Theoretical calculations

\begin{tabular}{|c|c|c|c|c|c|c|c|c|c|}
\hline \multirow{2}{*}{$\begin{array}{l}\text { Sl } \\
\text { No }\end{array}$} & \multirow[t]{2}{*}{ Grade of concrete } & \multirow[t]{2}{*}{$\%$ of fiber } & \multirow[t]{2}{*}{$\mathrm{D} / \mathrm{T}$ ratio } & \multirow[t]{2}{*}{$\mathrm{L} / \mathrm{D}$ ratio } & \multirow[t]{2}{*}{ Exp load, kN } & \multicolumn{4}{|c|}{ Axial Load Carrying Capacity $(\mathrm{kN})$} \\
\hline & & & & & & ACI & BS & EC-4 & CCECS \\
\hline 1 & \multirow{6}{*}{$\begin{array}{l}3 \\
0 \\
\exists \\
0 \\
0 \\
0\end{array}$} & - & 10.53 & 10 & 112 & 76.04 & 86.50 & 95.05 & 95.36 \\
\hline 2 & & - & 10.53 & 12 & 109 & 76.04 & 86.50 & 95.05 & 95.36 \\
\hline 3 & & - & 10.53 & 14 & 103 & 76.04 & 86.50 & 95.05 & 95.36 \\
\hline 4 & & - & 13.25 & 10 & 135 & 97.73 & 111.17 & 122.16 & 122.51 \\
\hline 5 & & - & 13.25 & 12 & 128 & 97.73 & 111.17 & 122.16 & 122.51 \\
\hline 6 & & - & 13.25 & 14 & 124 & 97.73 & 111.17 & 122.16 & 122.51 \\
\hline 7 & M 20 & 0 & 10.53 & 10 & 130 & 85.00 & 92.42 & 107.26 & 103.15 \\
\hline 8 & M20 & 0.2 & 10.53 & 10 & 136 & 86.81 & 93.62 & 109.92 & 107.52 \\
\hline 9 & M20 & 0.3 & 10.53 & 10 & 139 & 87.43 & 93.89 & 110.83 & 108.46 \\
\hline 10 & M20 & 0.4 & 10.53 & 10 & 133 & 88.00 & 94.41 & 111.68 & 110.28 \\
\hline 11 & M20 & 0 & 10.53 & 12 & 127 & 85.00 & 92.42 & 108.22 & 96.89 \\
\hline 12 & M20 & 0.2 & 10.53 & 12 & 133 & 86.81 & 93.62 & 110.89 & 100.99 \\
\hline 13 & M20 & 0.3 & 10.53 & 12 & 137 & 87.43 & 93.89 & 111.79 & 101.88 \\
\hline 14 & M20 & 0.4 & 10.53 & 12 & 130 & 88.00 & 94.41 & 112.64 & 103.59 \\
\hline 15 & M20 & 0 & 10.53 & 14 & 125 & 85.00 & 92.42 & 108.22 & 91.38 \\
\hline 16 & M20 & 0.2 & 10.53 & 14 & 130 & 86.81 & 93.62 & 110.89 & 95.25 \\
\hline 17 & M20 & 0.3 & 10.53 & 14 & 136 & 87.43 & 93.89 & 111.79 & 96.08 \\
\hline 18 & M20 & 0.4 & 10.53 & 14 & 129 & 88.00 & 94.41 & 112.64 & 97.70 \\
\hline 19 & M20 & $0 \%$ & 13.25 & 10 & 165 & 113.31 & 121.48 & 145.07 & 142.20 \\
\hline 20 & M20 & 0.2 & 13.25 & 10 & 171 & 116.46 & 123.56 & 149.70 & 149.19 \\
\hline 21 & M20 & 0.3 & 13.25 & 10 & 176 & 117.53 & 124.02 & 151.28 & 150.70 \\
\hline 22 & M20 & 0.4 & 13.25 & 10 & 168 & 118.53 & 124.93 & 152.75 & 153.63 \\
\hline 23 & M20 & 0 & 13.25 & 12 & 161 & 113.31 & 121.48 & 145.07 & 133.57 \\
\hline 24 & M20 & 0.2 & 13.25 & 12 & 167 & 116.46 & 123.56 & 149.70 & 140.14 \\
\hline 25 & M20 & 0.3 & 13.25 & 12 & 169 & 117.53 & 124.02 & 151.28 & 141.56 \\
\hline 26 & M20 & 0.4 & 13.25 & 12 & 163 & 118.53 & 124.93 & 152.75 & 144.31 \\
\hline 27 & M20 & 0 & 13.25 & 14 & 158 & 113.31 & 121.48 & 145.07 & 125.97 \\
\hline 28 & M20 & 0.2 & 13.25 & 14 & 162 & 116.46 & 123.56 & 149.70 & 132.16 \\
\hline 29 & M20 & 0.3 & 13.25 & 14 & 166 & 117.53 & 124.02 & 151.28 & 133.50 \\
\hline 30 & M20 & 0.4 & 13.25 & 14 & 160 & 118.53 & 124.93 & 152.75 & 136.10 \\
\hline 31 & M30 & 0 & 10.53 & 10 & 140 & 88.76 & 94.91 & 112.79 & 112.00 \\
\hline 32 & M30 & 0.2 & 10.53 & 10 & 146 & 89.60 & 95.47 & 114.03 & 113.87 \\
\hline 33 & M30 & 0.3 & 10.53 & 10 & 149 & 90.17 & 95.85 & 114.87 & 115.13 \\
\hline 34 & M30 & 0.4 & 10.53 & 10 & 142 & 90.88 & 96.32 & 115.91 & 116.67 \\
\hline 35 & M30 & 0 & 10.53 & 12 & 137 & 88.76 & 94.91 & 113.75 & 105.20 \\
\hline 36 & M30 & 0.2 & 10.53 & 12 & 141 & 89.60 & 95.47 & 114.99 & 106.96 \\
\hline 37 & M30 & 0.3 & 10.53 & 12 & 146 & 90.17 & 95.85 & 115.83 & 108.14 \\
\hline 38 & M30 & 0.4 & 10.53 & 12 & 139 & 90.88 & 96.32 & 116.87 & $\begin{array}{l}109.59 \\
\end{array}$ \\
\hline 39 & M30 & 0 & 10.53 & 14 & 133 & 88.76 & 94.91 & 113.75 & 99.22 \\
\hline 40 & M30 & 0.2 & 10.53 & 14 & 138 & 89.60 & 95.47 & 114.99 & 100.87 \\
\hline 41 & M30 & 0.3 & 10.53 & 14 & 142 & 90.17 & 95.85 & 115.83 & 101.99 \\
\hline 42 & M30 & 0.4 & 10.53 & 14 & 135 & 90.88 & 96.32 & 116.87 & 103.35 \\
\hline 43 & M30 & 0 & 13.25 & 10 & 175 & 119.85 & 125.80 & 154.69 & 156.39 \\
\hline 44 & M30 & 0.2 & 13.25 & 10 & 180 & 121.31 & 126.77 & 156.83 & 159.40 \\
\hline 45 & M30 & 0.3 & 13.25 & 10 & 184 & 122.30 & 127.43 & 158.30 & 161.43 \\
\hline 46 & M30 & 0.4 & 13.25 & 10 & 178 & 123.54 & 128.25 & 160.11 & 163.92 \\
\hline 47 & M30 & 0 & 13.25 & 12 & 171 & 119.85 & 125.80 & 154.69 & 146.90 \\
\hline 48 & M30 & 0.2 & 13.25 & 12 & 178 & 121.31 & 126.77 & 156.83 & 149.73 \\
\hline 49 & M30 & 0.3 & 13.25 & 12 & 175 & 122.30 & 127.43 & 158.30 & 151.64 \\
\hline 50 & M30 & 0.4 & 13.25 & 12 & 173 & 123.54 & 128.25 & 160.11 & 153.97 \\
\hline 51 & M30 & 0 & 13.25 & 14 & 163 & 119.85 & 125.80 & 154.69 & 138.54 \\
\hline 52 & M30 & 0.2 & 13.25 & 14 & 169 & 121.31 & 126.77 & 156.83 & 141.21 \\
\hline 53 & M30 & 0.3 & 13.25 & 14 & 172 & 122.30 & 127.43 & 158.30 & 143.01 \\
\hline 54 & M30 & 0.4 & 13.25 & 14 & 165 & 123.54 & 128.25 & 160.11 & 145.21 \\
\hline
\end{tabular}


From above results we can concluded that

$>$ load carrying capacity of the CFST column increases with decreasing $1 / \mathrm{d}$ ratio.

$>$ As $\mathrm{D} / \mathrm{T}$ ratio enhances the load bearing capacity of the member increases.

$>$ The columns of smaller slenderness ratio Columns with greater slenderness ratio fail by overall buckling.

$>$ From the analytical results that the decrease in L/D ratio increases the capacity of the CFST section.

$>$ As grade of concrete increases the strength of the CFST member.

\subsection{Graphical Representation of Theoretical}

\section{Calculations}

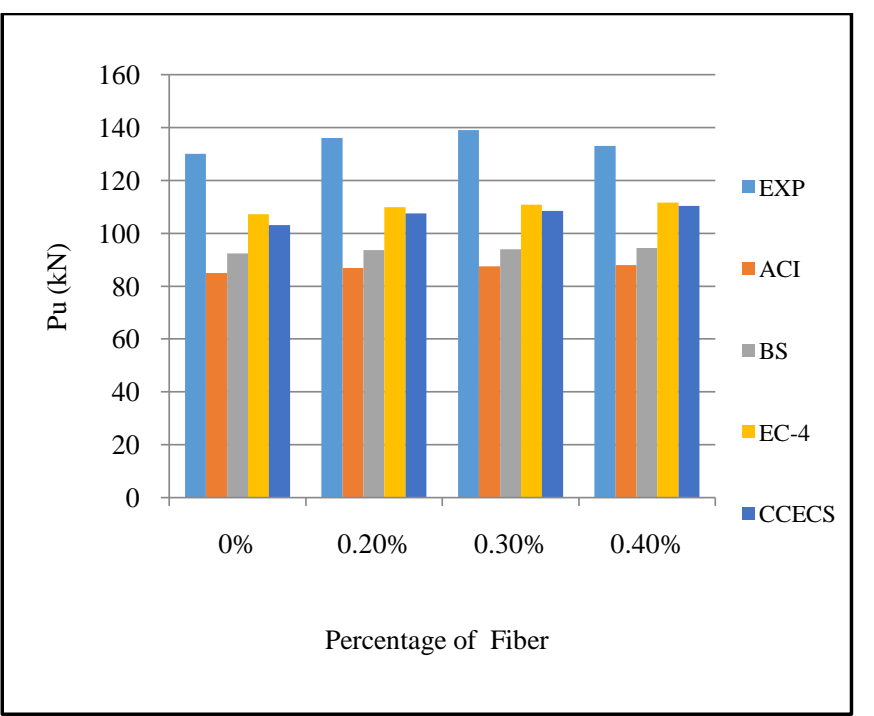

Chart 2: Variation of Pu for different Percentage of fiber $\mathrm{d} 1=33.7 \mathrm{~mm} \mathrm{~L} / \mathrm{D}$ ratio $=10$ for $\mathrm{M} 20$ grade of concrete.

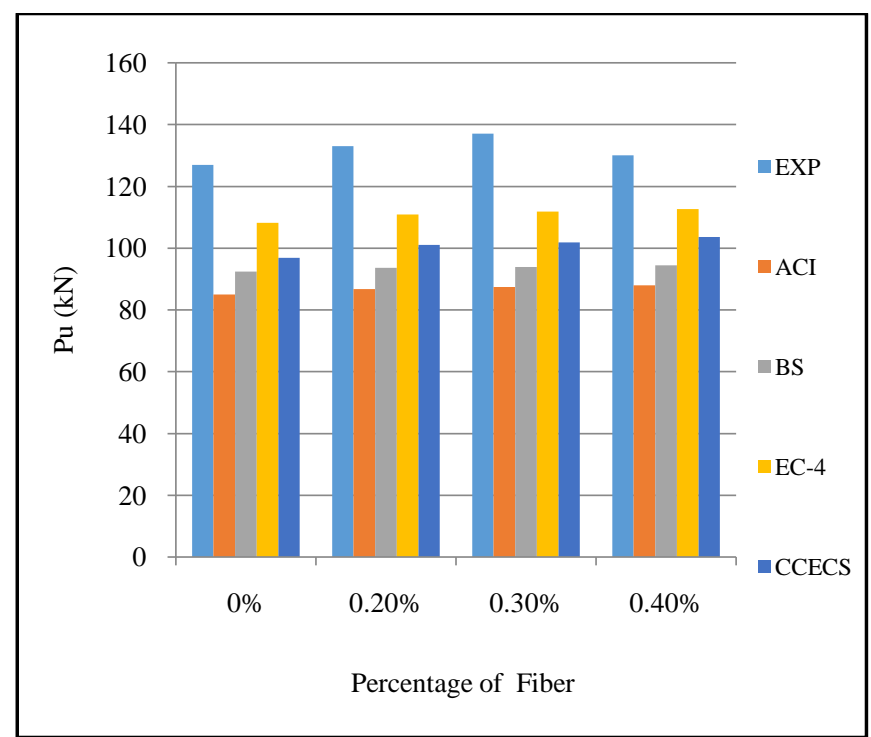

Chart 3: Variation of $\mathrm{Pu}$ for different Percentage of fiber $\mathrm{d} 1=33.7 \mathrm{~mm} \mathrm{~L} / \mathrm{D}$ ratio $=12$ for $\mathrm{M} 20$ grade of concrete.

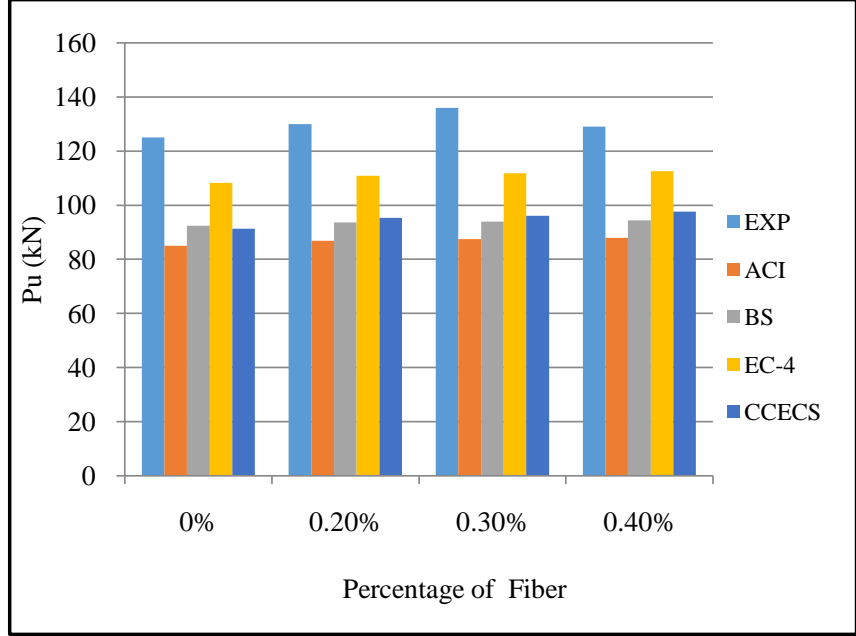

Chart 4: Variation of $\mathrm{Pu}$ for different Percentage of fiber $\mathrm{d} 1=33.7 \mathrm{~mm} \mathrm{~L} / \mathrm{D}$ ratio $=14$ for $\mathrm{M} 20$ grade of concrete.

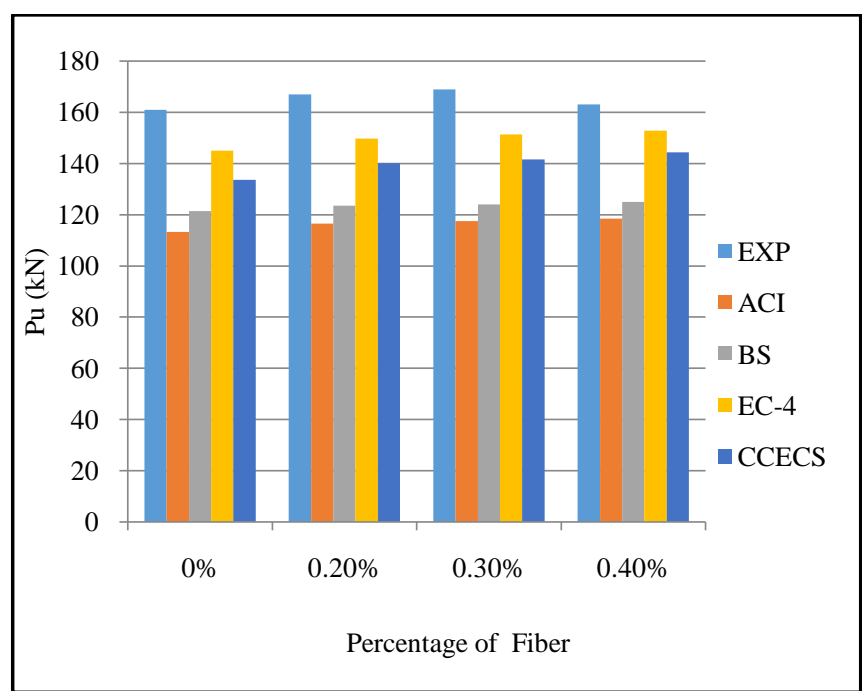

Chart 5: Variation of Pu for different Percentage of fiber $\mathrm{d} 2=42.4 \mathrm{~mm} \mathrm{~L} / \mathrm{D}$ ratio $=10$ for $\mathrm{M} 20$ grade of concrete.

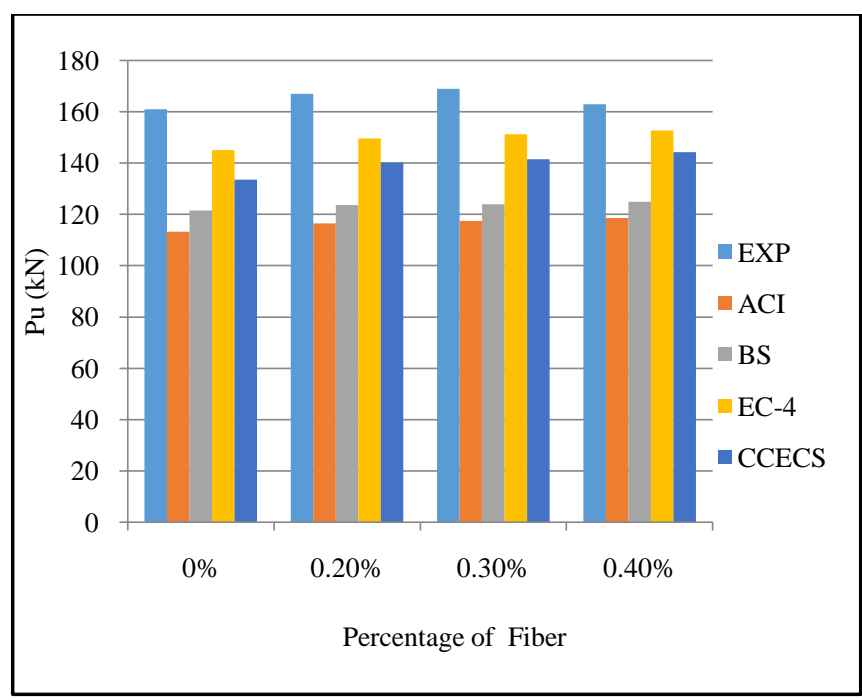

Chart 6: Variation of $\mathrm{Pu}$ for different Percentage of fiber $\mathrm{d} 2=42.4 \mathrm{~mm} \mathrm{~L} / \mathrm{D}$ ratio $=10$ for $\mathrm{M} 20$ grade of concrete. 


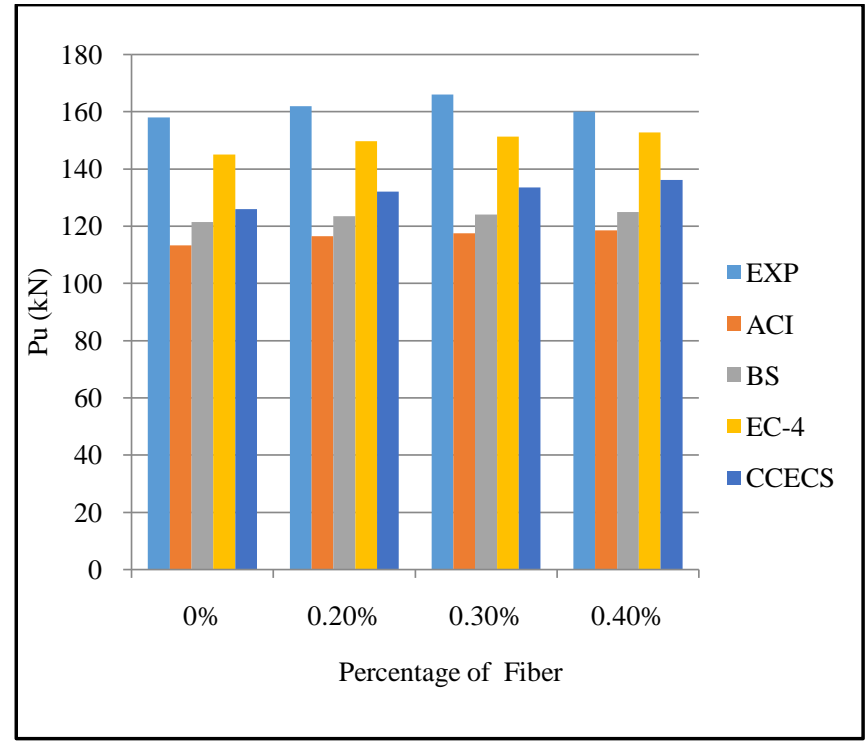

Chart 7: Variation of Pu for different Percentage of fiber $\mathrm{d} 2=42.4 \mathrm{~mm} \mathrm{~L} / \mathrm{D}$ ratio $=10$ for $\mathrm{M} 20$ grade of concrete.

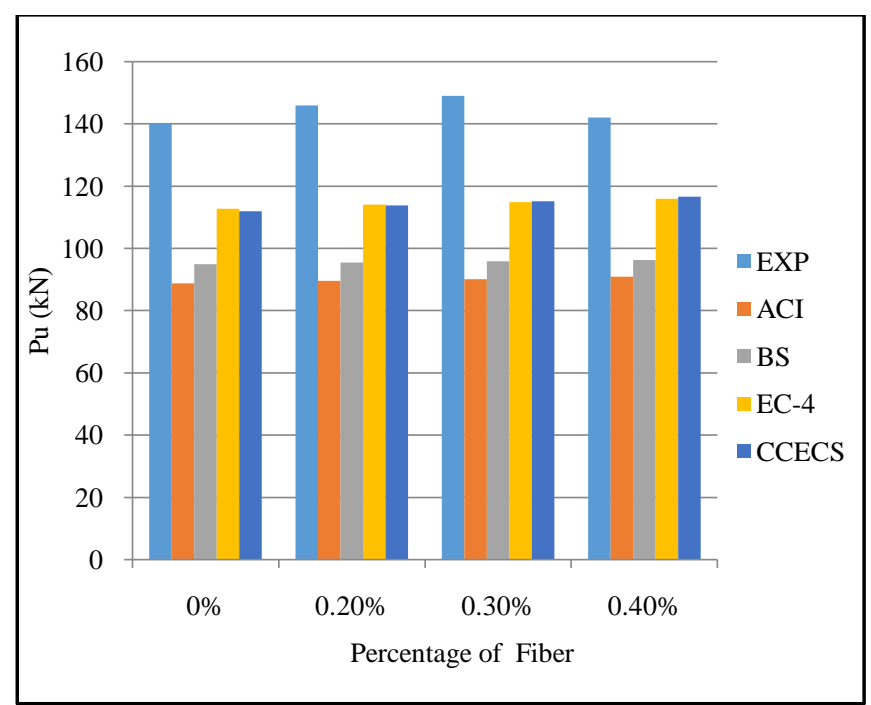

Chart 8: Variation of Pu for different Percentage of fiber $\mathrm{d} 1=33.7 \mathrm{~mm} \mathrm{~L} / \mathrm{D}$ ratio $=10$ for $\mathrm{M} 30$ grade of concrete.

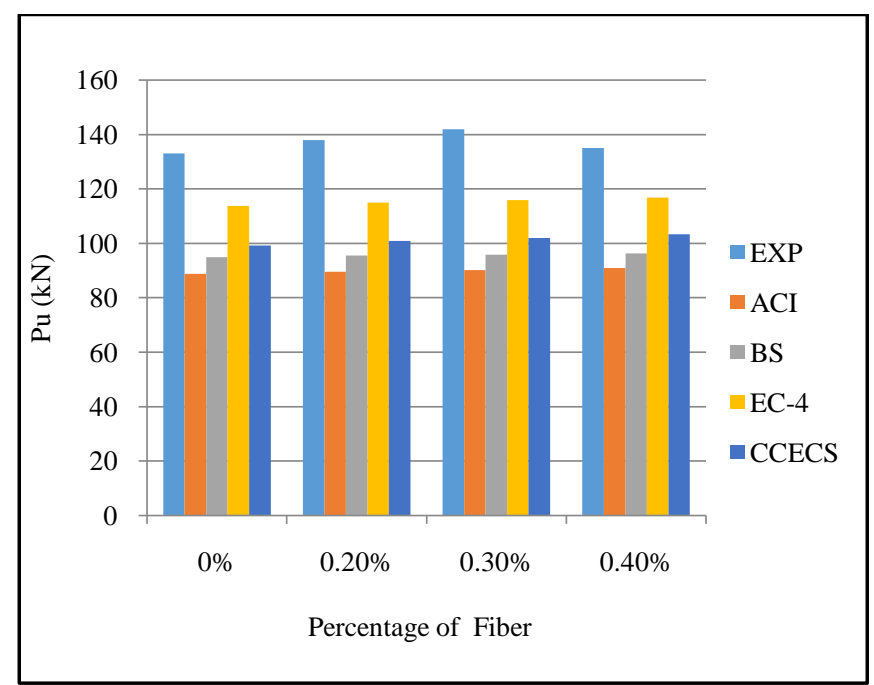

Chart 9: Variation of Pu for different Percentage of fiber $\mathrm{d} 12=42.4 \mathrm{~mm} \mathrm{~L} / \mathrm{D}$ ratio $=10$ for $\mathrm{M} 30$ grade of concrete.

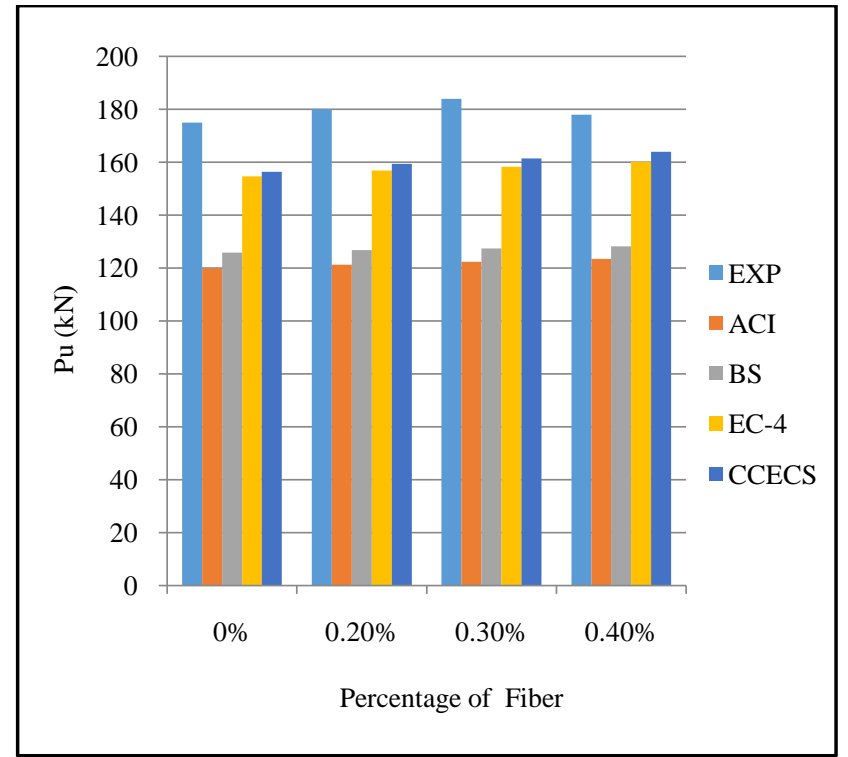

Chart 10: Variation of Pu for different Percentage of fiber $\mathrm{d} 12=42.4 \mathrm{~mm} \mathrm{~L} / \mathrm{D}$ ratio $=10$ for $\mathrm{M} 30$ grade of concrete.

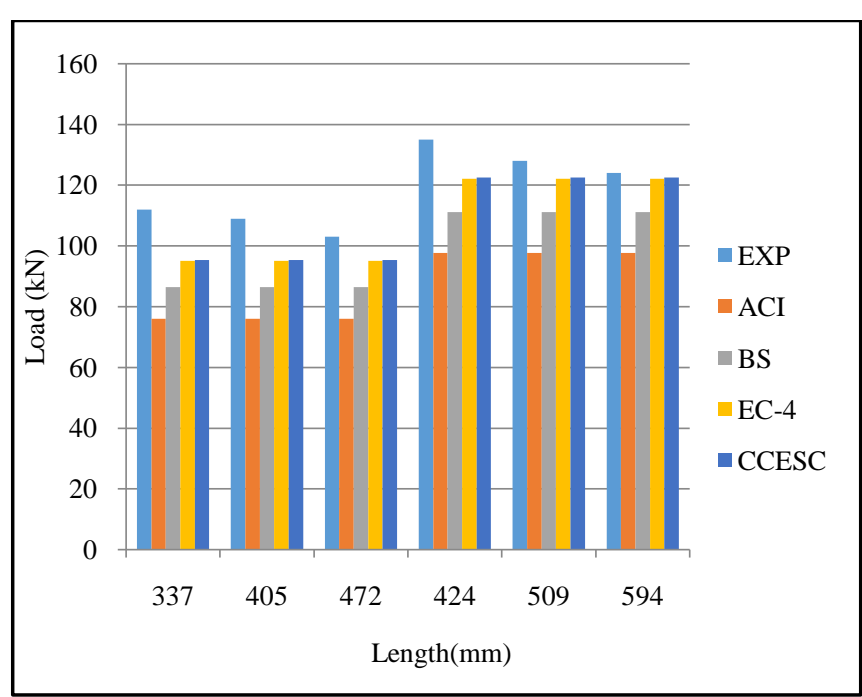

Chart 11: Variation of $\mathrm{Pu}$ for different length of hollow tubes

\section{FINITE ELEMENT ANALYSIS}

\subsection{Introduction}

The finite element method (FEM) is a numerical technique for finding approximate solutions to boundary value problems for partial differential equations. It is also referred to as finite element analysis (FEA). FEM helps in subdividing the large problem into smaller parts called finite elements.

The various considerations taken in the dicritization process is:

1. Type of elements

2. Size of elements

3. Location of nodes

4. Number of elements

7. Node numbering scheme

8. Automatic node generation 


\subsection{Load Carrying Capacity Using ABAQUS Software}

MODELLING

GEOMETRY

D

ELEMENT TYPE

$\sqrt{1}$

MATERIAL PROPERTIES

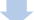

BOUNDRY CONDITION

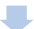

MESH DEFINITION

5

\section{ANALYSIS}

Fig 8: Modeling Procedure

\subsection{Material Specification}

\section{CONCRETE}

Grade of concrete

M20. Young's modulus E=22360 M pa

M30, Young's modulus E=27386.5 M pa

Poison ratio $=0.2$

Density $=2400 \mathrm{~kg} / \mathrm{m} 3$

\section{STEEL}

Grade of steel: $\mathrm{Fe}=250 \mathrm{M}$ pa

Young's modulus $=210000 \mathrm{M}$ pa

Poison ratio $=0.3$

Density $=7850 \mathrm{~kg} / \mathrm{m} 3$

\subsection{Boundary Condition}

The bottom ends are fixed, for fixed condition the degree of freedom is 6 i.e. 3 degree of rotation, 3 degree of displacement.

\subsection{Modeling and Analysis Using Hypermesh and}

\section{Abacus}

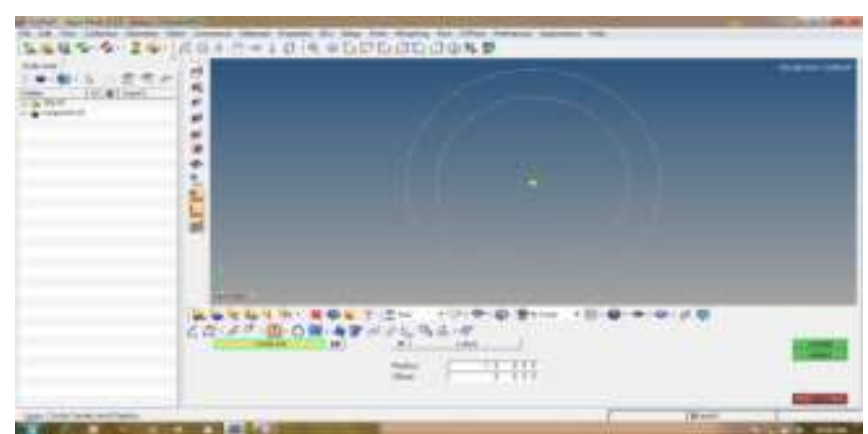

Fig 9: Creating CFST model

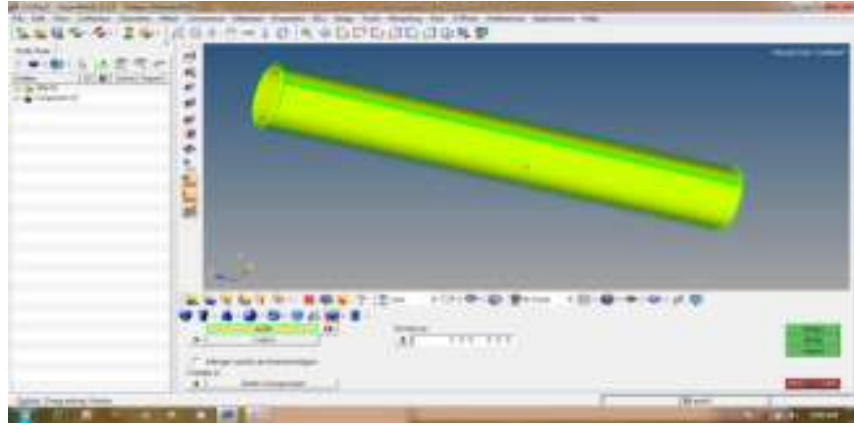

Fig 10: Segregation Of Elements

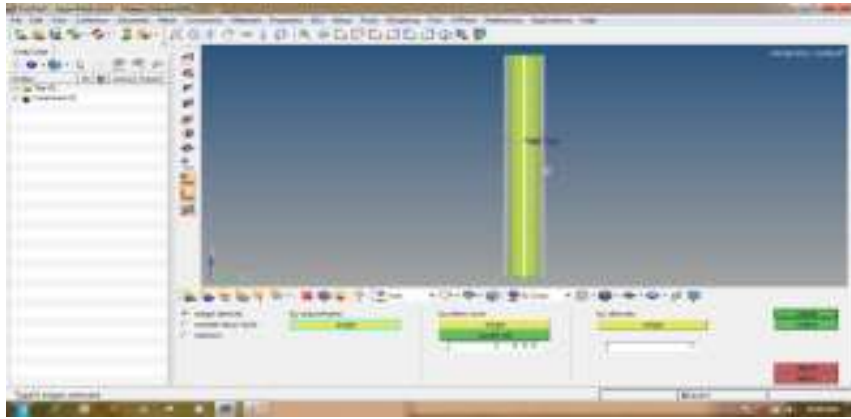

Fig 11: Meshing of CFST column

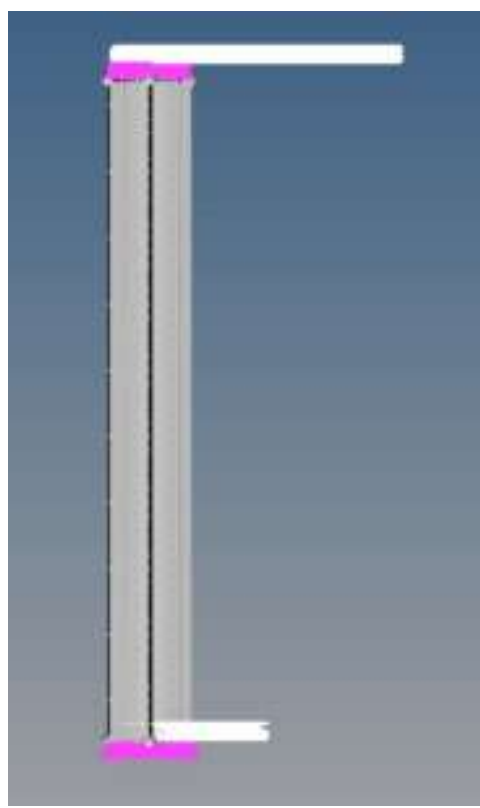

Fig12: applying the load

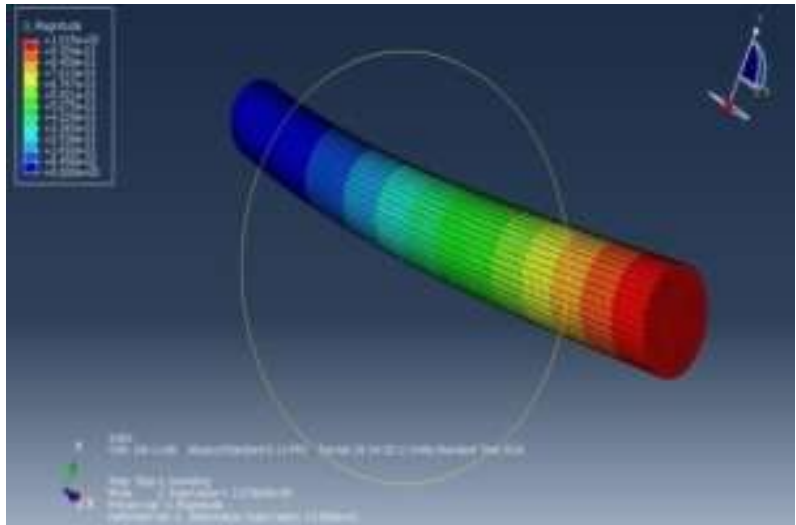

Fig 13: Mode 1 deformation 


\subsection{Analysis Result}

\begin{tabular}{|c|c|c|c|c|c|c|c|c|}
\hline Exp no & $\begin{array}{l}\text { Percentage } \\
\text { of Fiber }\end{array}$ & Grade & $\begin{array}{l}\text { Length, } \\
\mathrm{mm}\end{array}$ & $\begin{array}{l}\text { Area, } \mathrm{mm} \\
2\end{array}$ & $\begin{array}{l}\text { (ANALYSIS) } \\
\text { PU (kN) }\end{array}$ & $\begin{array}{l}\text { Exp Load, } \\
\text { PU( kN) }\end{array}$ & $\begin{array}{l}\text { Percentage } \\
\text { ERROR }\end{array}$ & $\begin{array}{l}\text { Grade of } \\
\text { concrete }\end{array}$ \\
\hline 1 & & - & 337 & 306.62 & 146 & 112 & 12.5 & \multirow[t]{6}{*}{ HOLLOW } \\
\hline 2 & & - & 405 & 306.62 & 142 & 109 & 11.9 & \\
\hline 3 & & - & 472 & 306.62 & 135 & 103 & 16.5 & \\
\hline 4 & & - & 424 & 394.08 & 163 & 135 & 3.7 & \\
\hline 5 & & - & 509 & 394.08 & 157 & 128 & 7.0 & \\
\hline 6 & & - & 594 & 394.08 & 142 & 121 & 12.4 & \\
\hline 7 & 0 & M20 & 337 & 891.96 & 183 & 130 & 13.8 & M20 \\
\hline 8 & 0.2 & M20 & 337 & 891.96 & 198 & 136 & 13.2 & M20 \\
\hline 9 & 0.3 & M20 & 337 & 891.96 & 212 & 139 & 13.7 & M20 \\
\hline 10 & 0.4 & M20 & 337 & 1411.95 & 188 & 133 & 13.5 & M20 \\
\hline 11 & 0 & M20 & 405 & 1411.95 & 176 & 127 & 15.0 & M20 \\
\hline 12 & 0.2 & M20 & 405 & 1411.95 & 189 & 133 & 13.5 & M20 \\
\hline 13 & 0.3 & M20 & 405 & 891.96 & 209 & 137 & 13.1 & M20 \\
\hline 14 & 0.4 & M20 & 405 & 891.96 & 181 & 130 & 13.8 & M20 \\
\hline 15 & 0 & M20 & 472 & 891.96 & 171 & 125 & 12.8 & M20 \\
\hline 16 & 0.2 & M20 & 472 & 1411.95 & 181 & 130 & 13.8 & M20 \\
\hline 17 & 0.3 & M20 & 472 & 1411.95 & 198 & 136 & 10.3 & M20 \\
\hline 18 & 0.4 & M20 & 472 & 1411.95 & 176 & 129 & 10.9 & M20 \\
\hline 19 & 0 & M20 & 424 & 891.96 & 232 & 165 & 6.1 & M20 \\
\hline 20 & 0.2 & M20 & 424 & 891.96 & 258 & 171 & 10.5 & M20 \\
\hline 21 & 0.3 & M20 & 424 & 891.96 & 273 & 176 & 9.7 & M20 \\
\hline 22 & 0.4 & M20 & 424 & 1411.95 & 265 & 168 & 10.1 & M20 \\
\hline 23 & 0 & M20 & 509 & 1411.95 & 239 & 161 & 6.8 & M20 \\
\hline 24 & 0.2 & M20 & 509 & 1411.95 & 258 & 167 & 8.4 & M20 \\
\hline 25 & 0.3 & M20 & 509 & 891.96 & 262 & 169 & 11.2 & M20 \\
\hline 26 & 0.4 & M20 & 509 & 891.96 & 241 & 163 & 9.2 & M20 \\
\hline 27 & 0 & M20 & 594 & 891.96 & 230 & 158 & 5.7 & M20 \\
\hline 28 & 0.2 & M20 & 594 & 1411.95 & 251 & 162 & 8.6 & M20 \\
\hline 29 & 0.3 & M20 & 594 & 1411.95 & 258 & 166 & 7.8 & M20 \\
\hline 30 & 0.4 & M20 & 594 & 1411.95 & 226 & 160 & 7.5 & M20 \\
\hline 31 & 0 & M30 & 337 & 891.96 & 218 & 140 & 11.4 & M20 \\
\hline 32 & 0.2 & M30 & 337 & 891.96 & 222 & 146 & 11.6 & M30 \\
\hline 33 & 0.3 & M30 & 337 & 891.96 & 239 & 149 & 12.1 & M30 \\
\hline 34 & 0.4 & M30 & 337 & 1411.95 & 210 & 142 & 12.7 & M30 \\
\hline 35 & 0 & M30 & 405 & 1411.95 & 198 & 137 & 8.0 & M30 \\
\hline 36 & 0.2 & M30 & 405 & 1411.95 & 211 & 141 & 12.8 & M30 \\
\hline 37 & 0.3 & M30 & 405 & 891.96 & 226 & 146 & 11.0 & M30 \\
\hline 38 & 0.4 & M30 & 405 & 891.96 & 189 & 139 & 12.2 & M30 \\
\hline 39 & 0 & M30 & 472 & 891.96 & 192 & 133 & 7.5 & M30 \\
\hline 40 & 0.2 & M30 & 472 & 1411.95 & 216 & 138 & 10.1 & M30 \\
\hline 41 & 0.3 & M30 & 472 & 1411.95 & 229 & 142 & 9.9 & M30 \\
\hline 42 & 0.4 & M30 & 472 & 1411.95 & 190 & 135 & 9.6 & M30 \\
\hline 43 & 0 & M30 & 424 & 891.96 & 259 & 175 & 3.4 & M30 \\
\hline 44 & 0.2 & M30 & 424 & 891.96 & 265 & 180 & 8.3 & M30 \\
\hline 45 & 0.3 & M30 & 424 & 891.96 & 299 & 184 & 9.2 & M30 \\
\hline 46 & 0.4 & M30 & 424 & 1411.95 & 259 & 178 & 8.4 & M30 \\
\hline 47 & 0 & M30 & 509 & 1411.95 & 256 & 171 & 4.7 & M30 \\
\hline 48 & 0.2 & M30 & 509 & 1411.95 & 269 & 178 & 6.2 & M30 \\
\hline 49 & 0.3 & M30 & 509 & 891.96 & 272 & 175 & 12.6 & M30 \\
\hline 50 & 0.4 & M30 & 509 & 891.96 & 223 & 173 & 4.6 & M30 \\
\hline 51 & 0 & M30 & 594 & 891.96 & 234 & 163 & 4.9 & M30 \\
\hline 52 & 0.2 & M30 & 594 & 1411.95 & 246 & 169 & 7.7 & M30 \\
\hline 53 & 0.3 & M30 & 594 & 1411.95 & 258 & 172 & 9.9 & M30 \\
\hline 54 & 0.4 & M30 & 594 & 1411.95 & 214 & 165 & 8.5 & M30 \\
\hline
\end{tabular}




\subsection{Graphical Representation of Experimental and}

\section{Analytical Results}

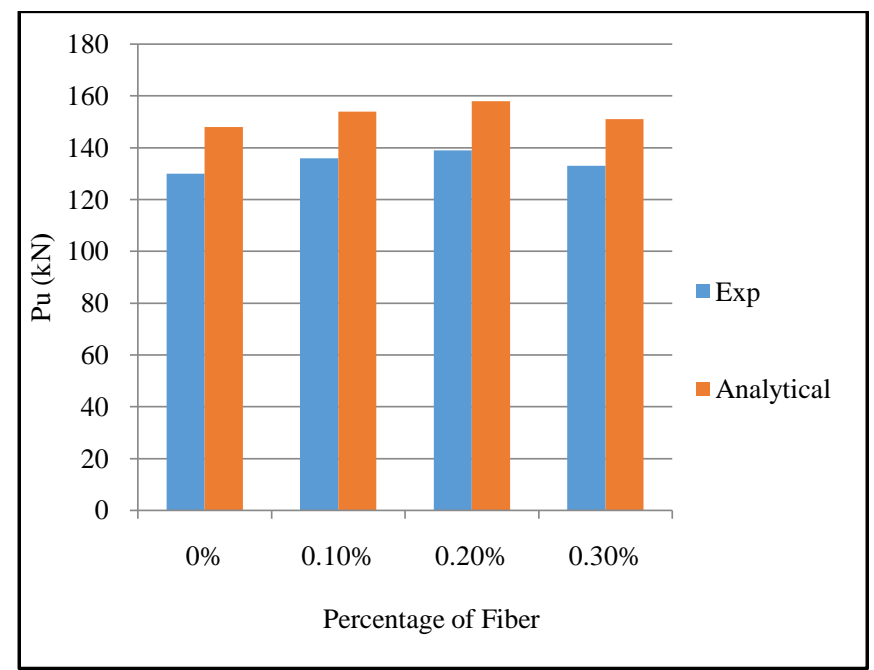

Chart 12: Variation of $\mathrm{Pu}$ for different Percentage of fiber $\mathrm{d} 1=33.7 \mathrm{~mm} \mathrm{~L} / \mathrm{D}$ ratio $=10$ for $\mathrm{M} 20$ grade of concrete

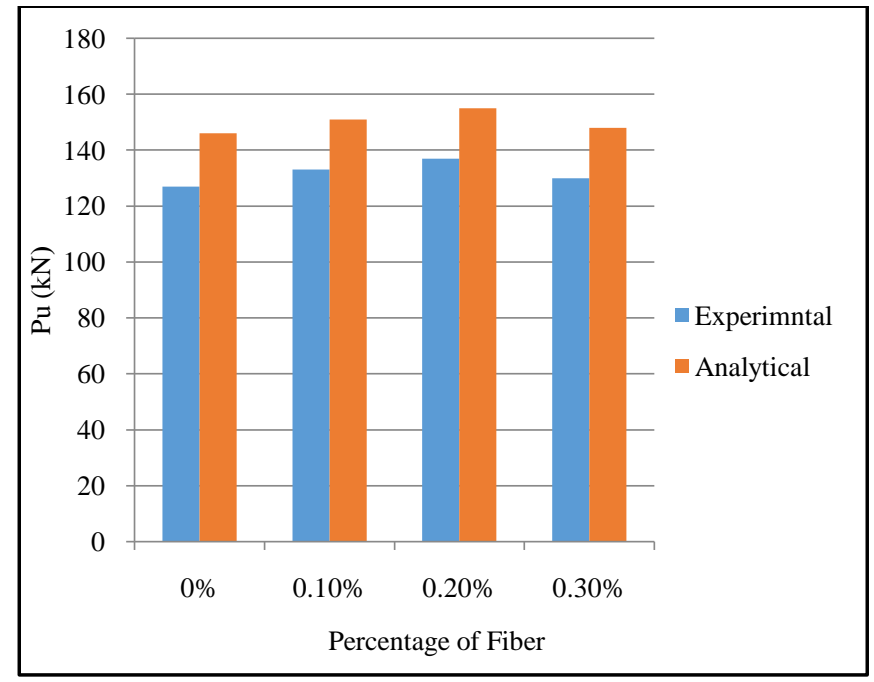

Chart 13: Variation of $\mathrm{Pu}$ for different Percentage of fiber $\mathrm{d} 1=33.7 \mathrm{~mm} \mathrm{~L} / \mathrm{D}$ ratio $=12$ for $\mathrm{M} 20$ grade of concrete

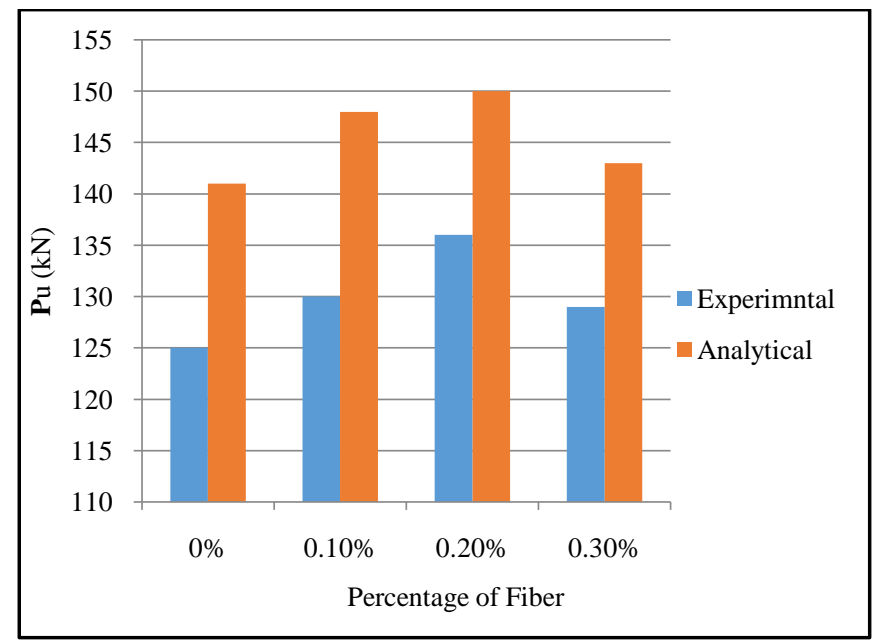

Chart 13: Variation of $\mathrm{Pu}$ for different Percentage of fiber $\mathrm{d} 1=33.7 \mathrm{~mm} \mathrm{~L} / \mathrm{D}$ ratio $=14$ for $\mathrm{M} 20$ grade of concrete

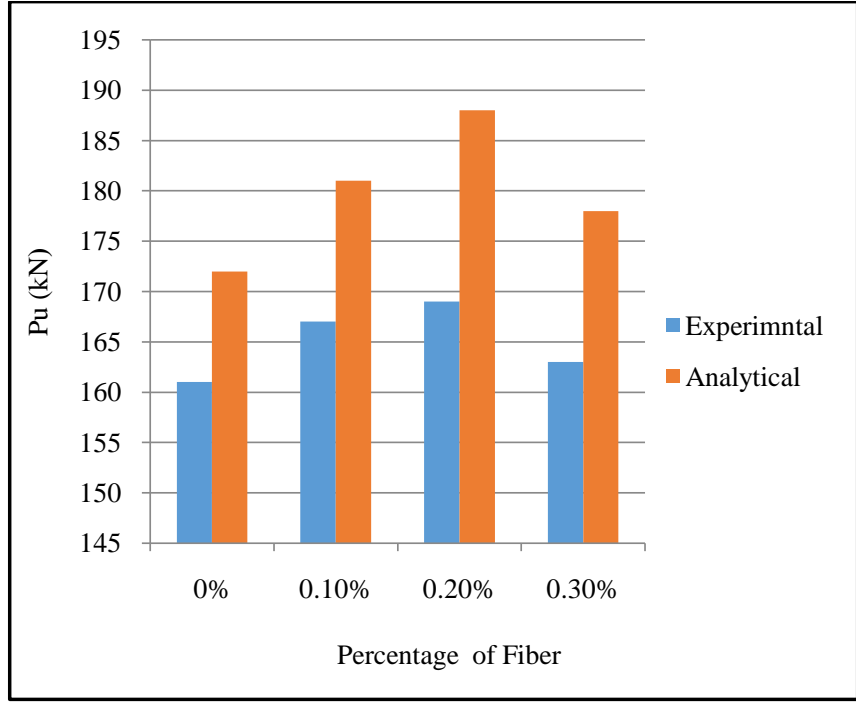

Chart 13: Variation of $\mathrm{Pu}$ for different Percentage of fiber $\mathrm{d} 2=42.4 \mathrm{~mm} \mathrm{~L} / \mathrm{D}$ ratio $=14$ for $\mathrm{M} 20$ grade of concrete

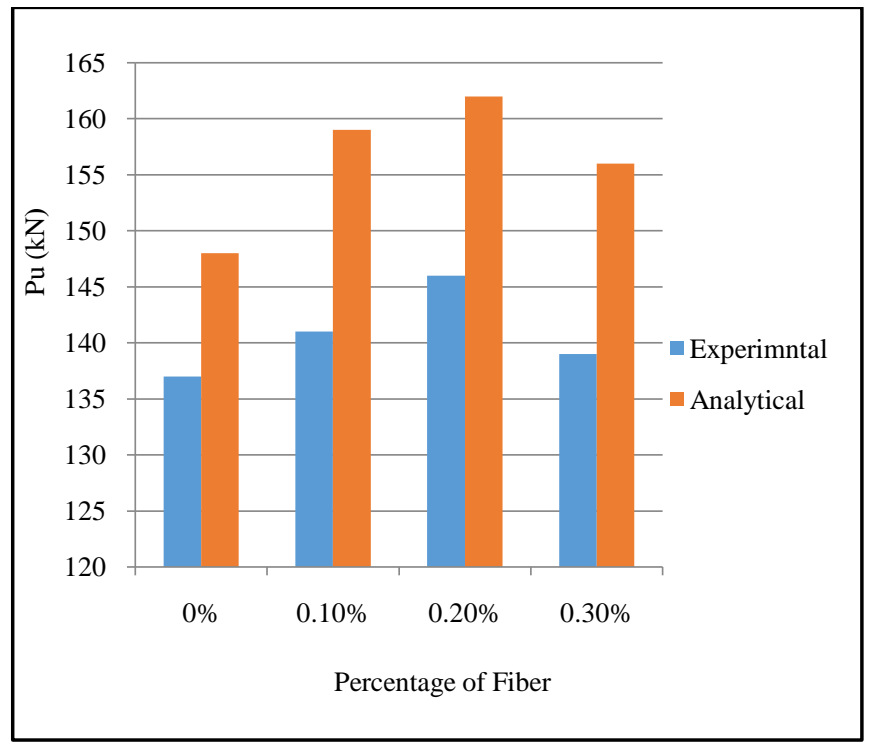

Chart 14: Variation of Pu for different Percentage of fiber $\mathrm{d} 1=33.7 \mathrm{~mm} \mathrm{~L} / \mathrm{D}$ ratio $=12$ for $\mathrm{M} 30$ grade of concrete

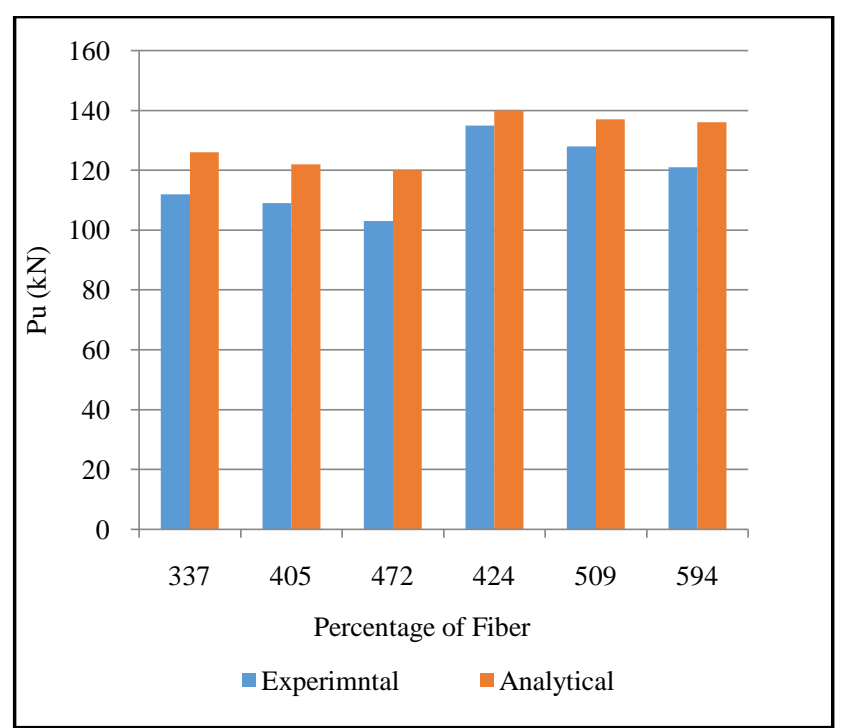




\section{CONCLUSION}

1. Load carrying capacity $(\mathrm{Pu})$ decreases with increase in L/D ratio for a given thickness and a given grade of concrete.

2. Load carrying capacity $(\mathrm{Pu})$ increases with increase in diameter of steel tube for a given L/D ratio, grade of concrete and a given thickness.

3. Load carrying capacity $(\mathrm{Pu})$ increases with increase in diameter for addition of fiber up to $0.3 \%$, a slight decrease in $\mathrm{Pu}$ is observed for $0.4 \%$ of fiber.

4. The deformation decreases with increase in diameter for a given L/D ratio, grade of concrete and thickness of tube.

5. The experimental results closely match with results obtained from various codal formulae.

\section{REFERENCES}

[1]. Elremaily, A., and Azizinamini, A._2002_. "Behaviour and strength of circular concrete-filled tube columns." $J$. Constr. Steel Res., 58, 1567-1591.

[2]. Goode, C. D., and Lam, D. _2008_. "Concrete-filled tube columns-Tests compared with Eurocode 4." Proc., Engineering Foundation Conf. On Composite Construction [3]. Cederwall K., Engstorm B., and Guauers M., (1990), "High strength concrete used in composite columns", Proceedings of High strength concrete, 2nd International Symposium, Detroit, 195-214 (ACI-SP 121)

[5]. Lu YQ, Kennedy DJL, (1994), "The flexural behaviour of concrete filled hollow structural sections", Canadian Journal of Civil Engineering, 21(1); 11-130.

[6]. Pan YH., Zhong ST., (1990), "Constitutive relation of concrete filled steel tube under tension", Ind. 